\section{Heparin, gepuffertes}

W. G. Guder

München, Deutschland

Synonym(e) Elektrolyt-stabilisierte Heparinlösung; Elektrolyt-balancierte Heparinlösung

Englischer Begriff electrolyte balanced heparin solution; buffered heparin solution

Definition Bei Anwendung von Heparin zur Gewinnung von Blutproben für die Messung von Elektrolyten und Blutgasen im Vollblut verwendete Heparinlösung, die in Zusammensetzung und $\mathrm{pH}-$ Wert normalem Plasma angepasst ist.

Beschreibung Bei Gewinnung von arteriellem, venösem oder kapillärem Blut für die Blutgas- und Elektrolytanalyse wird oft gelöstes Heparin verwendet. Um eine Verdünnung der Messergebnisse oder Veränderungen durch atypischen $\mathrm{pH}$ der Heparinlösung zu vermeiden, wurden von der IFCC Heparinlösungen empfohlen, deren Lösungsmittel folgender Zusammensetzung entsprechen:

- Natrium: $120-150 \mathrm{mmol} / \mathrm{L}$

- Kalium: $3,5-4,5 \mathrm{mmol} / \mathrm{L}$

- Ionisiertes Calcium: $1,2-1,4 \mathrm{mmol} / \mathrm{L}$

- Chlorid: $100-130 \mathrm{mmol} / \mathrm{L}$

- $\mathrm{pH}: 6,0-8,0$

Die Lösung darf keine Calcium-bindenden Anionen enthalten (Phosphat, Carbonat, Sulfat).

\section{Literatur}

Burnett RW, Covington AK, Fogh-Andersen N et al (1995) Approved IFCC recommendations on whole blood sampling for simultaneous determination of $\mathrm{pH}$, blood gases and electrolytes. Eur J Clin Chem Clin Biochem 33:247-253 